\title{
Q-Monitoring startet in eine neue Runde - Teil 2
}

Ärztinnen und Ärzte wollen ihre Patienten optimal versorgen - sie engagieren sich bei ihrer Arbeit daher täglich für die Qualitätsentwicklung: mittels Fallbesprechungen, in Fortbildungen, durch die Überprüfung von Behandlungsstandards usw. Das Projekt Q-Monitoring will anhand von Zahlen das Spektrum an Qualitätsaktivitäten aufzeigen, die ambulant tätige Ärztinnen und Ärzte bereits heute leisten. Nach der erfolgreichen Pilotphase im Jahr 2010 beteiligen sich neu insgesamt zehn Fachgesellschaften am Q-Monitoring - und damit rund zwei Drittel der ambulant tätigen Ärzteschaft.

Die Zusammenarbeit mit den Fachgesellschaften ist der FMH beim Projekt Q-Monitoring ambulante Medizin CH besonders wichtig. Denn nur die Fachgesellschaften sind in der Lage, ihre fachspezifischen Qualitätsaktivitäten adäquat zu definieren. Deshalb möchte die Projektleitung auch die am Projekt beteiligten Fachgesellschaften zu Wort kommen lassen. In diesem Artikel berichten zwei Vertreter derjenigen Fachbereiche, die bereits 2010 die Pilotphase des Projekts unterstützt haben, über ihre Erfahrungen mit Q-Monitoring: die Psychiatrie und die Hausarztmedizin. In der SÄZ-Ausgabe vom 28. März 2012 werden

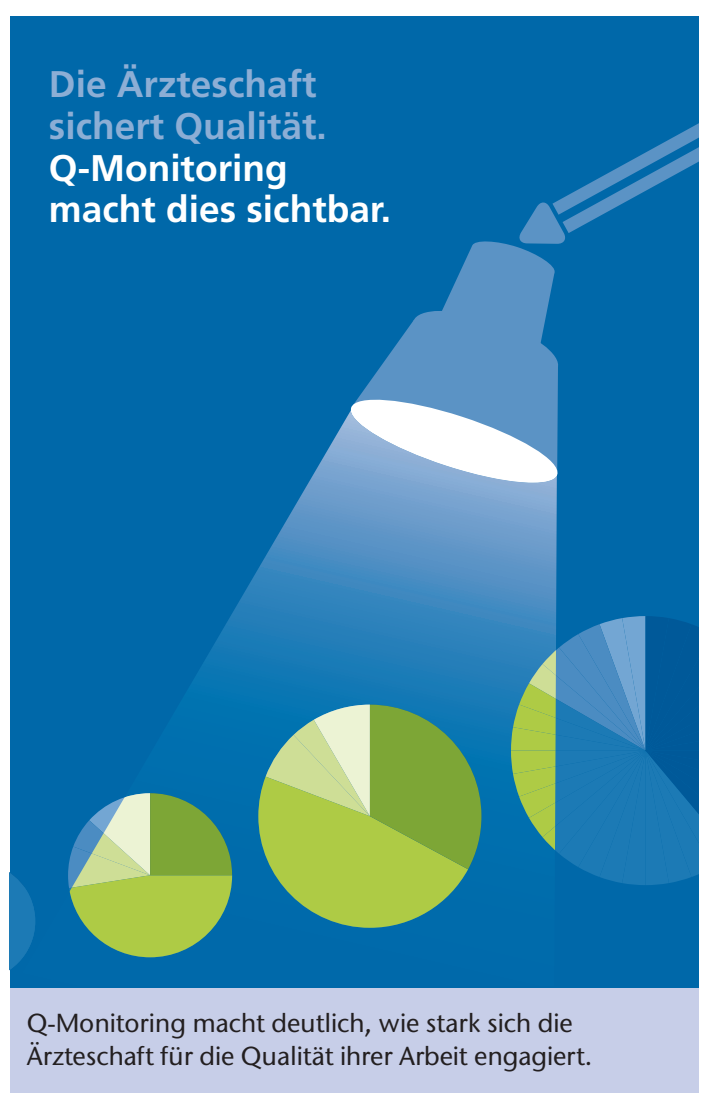

Vertreter der neu ins Projekt integrierten Fachrichtungen Gynäkologie und Geburtshilfe, Pneumologie, Anästhesiologie und Reanimation, Radiologie sowie ORL, Hals- und Gesichtschirurgie Stellung zum Projekt beziehen.

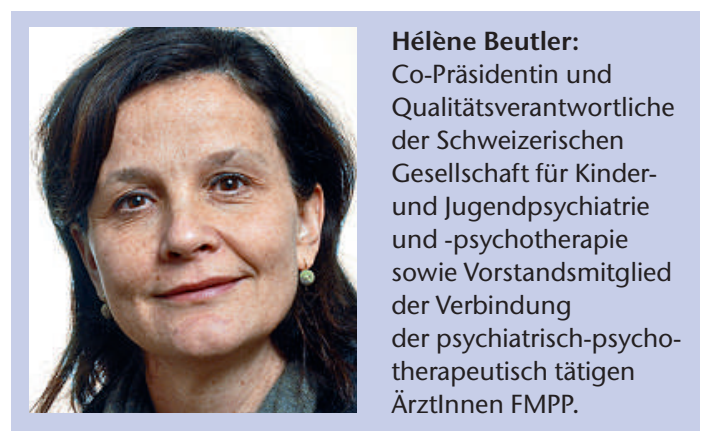

«Bereits 2010 haben die psychiatrischen Fachgesellschaften der Kinder- und Jugendpsychiatrie und -psychotherapie (SGKJPP) sowie der Psychiatrie und Psychotherapie (SGPP) die Chance genutzt und sich gemeinsam am Pilotprojekt Q-Monitoring beteiligt. Das Ziel, eine Bestandesaufnahme über die existierenden Qualitätsaktivitäten in der ambulanten psychiatrisch psychotherapeutischen Praxis zu erstellen und damit eine wertvolle Grundlage für die fachspezifische Qualitätsentwicklung zu bekommen, war ein wichtiger Grund für die Projektteilnahme. Aber auch das Bedürfnis, unser tägliches Engagement für eine qualitativ hochstehende Berufstätigkeit gegen aussen sichtbarer zu machen, war mit ausschlaggebend dafür, dass wir das Projekt unterstützen.

Das während der Pilotphase eingetroffene Feedback der teilnehmenden Psychiaterinnen und Psychiater hat uns motiviert, uns auch 2012 für die Erhebung zu engagieren. Während eines guten halben Jahrs haben wir zusammen mit der Abteilung Daten, Demographie und Qualität der FMH sowie weiteren beteiligten Fachgesellschaften die Erhebungsinstrumente aufgrund der Rückmeldungen verbessert und 
weiterentwickelt. Es war uns ein Anliegen, den Fragebogen noch psychiatriespezifischer auszurichten. Die projektbezogenen Treffen mit ebenfalls am Projekt beteiligten anderen Fachgesellschaften hat uns aber auch eindrücklich gezeigt, dass viele der täglichen Qualitätsbemühungen fachübergreifend von Bedeutung sind. Die vertiefte Zusammenarbeit mit anderen Fachrichtungen im Bereich Qualität ermöglicht es uns, voneinander zu lernen und zu profitieren. Gespannt warten wir auf die Resultate der Q-Monitoring-Befragung und ermuntern alle ambulant tätigen Ärztinnen und Ärzte der Kinder- und Erwachsenenpsychiatrie, aber auch der übrigen teilnehmenden Fachrichtungen, sich an der Umfrage zu beteiligen!»

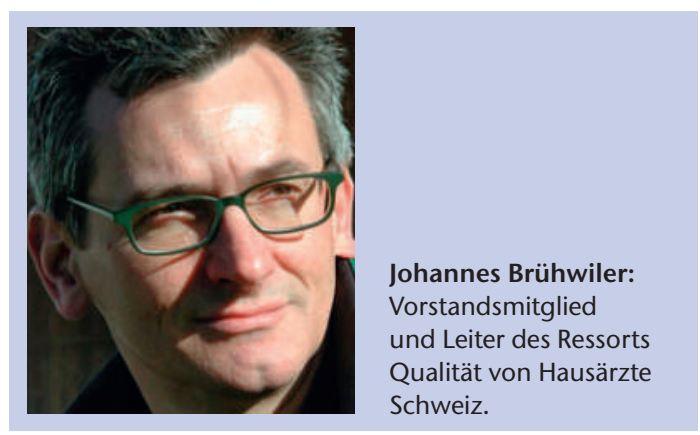

«Es lohnt sich, die Weiterführung des Projekts Q-Monitoring zu unterstützen: Davon sind der Verband Hausärzte Schweiz sowie die Vorstände der kinderund hausärztlichen Fachgesellschaften überzeugt! Denn Qualitätsentwicklung ist ein kontinuierlicher

\section{Start der Datenerfassung!}

Besitzen Sie einen der folgenden Facharzttitel?

- Allgemeine Innere Medizin*

- Anästhesiologie*

- Gynäkologie und Geburtshilfe*

- Kinder- und Jugendmedizin*

- Kinder- und Jugendpsychiatrie und -psychotherapie**

- Oto-Rhino-Laryngologie*

- Pneumologie**

- Psychiatrie und Psychotherapie**

- Radiologie*

* Umfrage läuft vom 8.2.-4.4. 2012

** Umfrage läuft vom 7.3.-2.5.2012

Dann bitten wir Sie, Ihre bereits heute geleisteten Qualitätsaktivitäten zu erfassen, selbstverständlich via freiwillige und vertrauliche Selbstdeklaration. Damit helfen Sie Ihrer Fachgesellschaft, die internen Qualitätsstrategien aufzubauen und weiterzuentwickeln. Gleichzeitig erhalten Sie die
Prozess. Und für die optimale Steuerung dieses Prozesses ist eine Übersicht über die aktuell getätigten Qualitätsaktivitäten für die Fachgesellschaften wichtig: Sie ist die Voraussetzung für eine qualitativ hochstehende haus- und kinderärztliche Versorgung. Ärztinnen und Ärzte, die den Fragebogen ausfüllen, erhalten eine individuelle, vertrauliche Rückmeldung über die bereits von ihnen geleisteten Aktivitäten. Dieser Überblick erlaubt jedem Einzelnen zu sehen, in welchen Bereichen er oder sie bereits viel leistet und wo allenfalls noch Optimierungspotential besteht. Aber auch für die standespolitischen Arbeiten der beteiligten Fachgesellschaften, der FMH sowie von Hausärzte Schweiz sind die Resultate zentral. Sie liefern eine wertvolle Grundlage für fundierte Argumente in der Qualitätsdiskussion mit unseren Partnern im Gesundheitswesen. Darum zählen wir auf eine rege Teilnahme an der Umfrage!

Da die Fragebögen seit der Pilotphase überarbeitet wurden, sind wir auf aktuelle Daten angewiesen. Deshalb bitten wir auch diejenigen Haus- und Kinderärzte um eine Teilnahme, die ihre Aktivitäten bereits während der Pilotphase 2010 registriert haben. Selbstverständlich wird die Vertraulichkeit der Daten auch dieses Jahr durch die Dateneingabe über das geschützte, persönliche Mitgliederportal myFMH strengstens gewahrt. MFE, die FMH sowie die hausund kinderärztlichen Fachgesellschaften SGAM, SGIM, SGP und Kinderärzte Schweiz (KIS) bedanken sich schon jetzt recht herzlich für Ihr Engagement!»

Möglichkeit, die von Ihnen geleisteten Qualitätsaktivitäten mit jenen des Gesamts ihrer Fachkolleginnen und -kollegen zu vergleichen. Mit Ihrer Teilnahme unterstützen Sie die Glaubwürdigkeit des ärztlichen Engagements für Qualitätsentwicklung und verbessern deren Transparenz entscheidend und nachhaltig!

Den Fragebogen finden Sie unter www.myfmh.ch. Falls Sie Fragen zum Projekt Q-Monitoring oder zum Fragebogen haben, geben wir gerne Auskunft: per E-Mail ddq[at]fmh.ch oder unter der Telefonnummer 03135911 11. Bei Fragen zu unserer Mitgliederplattform myFMH, insbesondere zur Registrierung, ist die Abteilung Dienstleistungen Mitglieder für Sie da: Telefon 03135912 59, E-Mail: myfmh[at]fmh.ch. Weitere Informationen zum Projekt Q-Monitoring finden Sie unter: www. $\mathrm{fmh}$.ch $\rightarrow$ Qualität $\rightarrow$ Q-Monitoring.

Die beteiligten Fachgesellschaften und die FMH danken Ihnen herzlich für Ihre Teilnahme! 